\title{
La construcción de las desigualdades salariales en la industria agroalimentaria
}

\author{
Rosario MORILLO BALADO* \\ Confederación Sindical de CC.OO. \\ lmorillo@ccoo.es
}

Recibido: 9-11-10

Aceptado: 23-6-11

\section{RESUMEN}

Este artículo expone los resultados de una investigación cualitativa sobre los procesos que influyen en la brecha salarial entre hombres y mujeres en la industria agroalimentaria. La infravaloración del trabajo realizado por las mujeres, su exclusión de los sectores y empleos con mejores perspectivas salariales así como sus reducidas trayectorias laborales son algunos de los mecanismos que contribuyen a mantener la desigualdad retributiva. El artículo indaga también en las dificultades que aparecen en la aplicación del principio de igual retribución por trabajos de igual valor como instrumento para garantizar la igualdad salarial.

Palabras clave: Desigualdad salarial, industria agroalimentaria, igual valor.

\section{The establishment of wage inequality in the food processing industry}

\begin{abstract}
This article highlights the results of a qualitative study into the processes which influence the wage gap between men and women in the food processing industry. The undervaluation of the work carried out by women, their exclusion from sectors and jobs with better salary prospects and reduced career expectations are some of the mechanisms which contribute to maintaining wage inequality. The article also looks into the problems that occur in applying the principle of equal pay for work of equal value as a means of guaranteeing wage equality.
\end{abstract}

Key words: pay gap, food processing industry, equal value.

\section{REFERENCIA NORMALIZADA}

Morillo Balado, R. (2011). La construcción de las desigualdades salariales en la industria agroalimentaria. Cuadernos de Relaciones Laborales, Vol. 29, núm. 2, p. 337-361.

SUMARIO: 1. Introducción. 2. Algunas causas de la desigualdad salarial. 3. Problemas con la aplicación del principio de igual retribución por trabajos de igual valor. 4. Metodología. 5. Resultados. 5.1. La exclu-

* Doctora en CC. Políticas y Sociología. Máster en Relaciones Laborales por la London School of Economics. Las opiniones expresadas en el artículo son responsabilidad de la autora y no comprometen a la organización para la que trabaja. 
sión de las mujeres de los mejores empleos y el peso de los estereotipos. 5.2. La infravaloración del trabajo de las mujeres. 5.3. El mayor control al que están sometidas las mujeres. 5.4. Perspectivas de cambio. 6 . Conclusiones. 7. Anexo. 8. Bibliografía.

\section{Introducción}

En España al igual que en otros países las mujeres ganan menos que los hombres. Las estadísticas oficiales ofrecen periódicamente datos sobre la existencia y dimensión del problema, pero no proporcionan información sobre los diferentes aspectos que se considera relevante comprender con el fin de poder diseñar soluciones (Jones y Torrie, 2009). Algunos de estos aspectos son los procesos que dan lugar a las diferencias salariales, la opinión de los principales actores o el papel que juegan las clasificaciones profesionales (Marini, 1989; Rubery et al., 2005). Estos objetivos son difíciles de aprehender con la metodología cuantitativa, más apropiada para la medición que para la comprensión. Por ello, este artículo adopta una metodología cualitativa con el fin de detectar los discursos y prácticas que se producen en torno a este problema.

El análisis se centra en un sector, la industria de alimentación y bebidas, significativo por el peso del empleo femenino y porque las diferencias salariales entre sexos son superiores a la media nacional. En esta actividad, el salario de las mujeres equivale al $66 \%$ del salario masculino. Es decir, las trabajadoras de ese sector ganan un $34 \%$ menos que los hombres, frente al $25 \%$ de diferencia que existe para el conjunto de la economía (Zarapuz, 2009). La industria de alimentación y bebidas -a la que denominaremos industria agroalimentaria a lo largo de este artículo- es además una rama de actividad importante en términos de empleo femenino: las asalariadas alcanzan el $39 \%$ de la mano de obra en el sector y, en el conjunto de la industria española, es la actividad que mayor número de mujeres emplea, 166.000. Estamos, por tanto, estudiando la desigualdad salarial en el colectivo de mujeres más numeroso de la industria española ${ }^{1}$.

Comenzamos el artículo examinando los diversos factores que se han utilizado para explicar la posición subordinada de las mujeres en el mercado de trabajo. Esto nos permite relacionar la brecha salarial con las tendencias más amplías que influyen en este problema, pues partimos de la premisa de que la desigualdad retributiva es el reflejo de los distintos tipos de situaciones desfavorables que sufren las mujeres en la esfera laboral. A continuación se tratan los problemas que otras investiga-

${ }^{1}$ En el trabajo de campo también se ha recogido información sobre el sector agrícola (código 01 de la CNAE de 2009), en concreto sobre el manipulado de frutas y hortalizas -es decir, su preparación para la venta en los mercados primarios- así como sobre el cultivo y recolección de flores. Pero sobre el sector agrícola, donde las mujeres representan el $26 \%$ del empleo asalariado, las estadísticas salariales no proporcionan datos sobre las diferencias retributivas entre sexos (Zarapuz, 2009). 
ciones han detectado en la aplicación del principio de igual salario por trabajo de igual valor como mecanismo para superar la desigualdad retributiva. La sección cuarta contiene las aclaraciones metodológicas. En la sección quinta se exponen los resultados de nuestra investigación agrupados en los procesos que contribuyen a mantener la desigualdad salarial. Al final de esa sección se abordan también las perspectivas de cambio, con especial referencia a los intentos de superar la infravaloración de las tareas femeninas a través del principio de igual valor. Concluye el artículo con una reflexión sobre las implicaciones de nuestros resultados en el logro de una mayor equidad retributiva.

\section{Algunas causas de la desigualdad salarial}

Se han apuntado diferentes factores para explicar la brecha salarial y la posición de las mujeres en el mercado laboral. En este epígrafe revisaremos los más relevantes para nuestra investigación: la segregación ocupacional, el acceso a la tecnología, la resistencia masculina, la movilidad ocupacional y la infravaloración de tareas.

La desigualdad salarial está relacionada con el papel de los empresarios y la segregación de los empleos por sexos, es decir, la concentración de las mujeres en un reducido número de ocupaciones y sectores (Joshi et al., 2007; Moore, 2007). Las empresas no consideran intercambiable la mano de obra femenina y masculina (Belt et al., 2002; Wajcman, 2000), sino que utilizan la diferente posición social de los sexos en su propio beneficio (Michon, 2005; Reich et al., 1973), creando puestos de trabajo específicos para las mujeres a los que se asignan salarios más bajos que a los hombres.

Otro factor que se ha utilizado para explicar la posición subordinada de las mujeres en el mercado de trabajo es su relación con la tecnología (Brynin, 2006; Caprile y Serrano, 2011; Cockburn, 1981). Las mujeres "continúan dándole a la tecla y a los hombres se les sigue adiestrando para comprender e intervenir en el manejo de la maquinaria" (Cockburn, 1992:93). El mayor acceso de los hombres a la tecnología se ha vinculado también con la formación de la identidad y la prevalencia de los estereotipos: la masculinidad se relaciona con las habilidades técnicas y la feminidad con la incompetencia en esta materia. Estos estereotipos pueden darse en las trabajadoras a la hora de elegir ocupación, en los empresarios en el momento de adjudicar puestos de trabajo o en los compañeros varones mediante estrategias de exclusión encaminadas a impedir el acceso de las mujeres (Lindsay, 2008; Peterson, 2007; Walby, 2011).

El papel de los hombres en el mantenimiento de los menores salarios femeninos ha sido objeto de diferentes investigaciones. Hartman (1976/1994) ofrece ejemplos de cómo históricamente los trabajadores varones han intentado limitar la participación de las mujeres en el mercado de trabajo o cuando esta se ha producido, mantenerla en una situación de inferioridad. Otras investigaciones han señalado que, cuando hombres y mujeres realizan tareas similares, los varones con frecuencia 
recurren a diferentes estrategias orientadas a elevar el estatus de sus tareas y puestos de trabajo (Simpson, 2004).

La desigualdad salarial entre hombres y mujeres está también relacionada con las reducidas trayectorias profesionales de las mujeres y con la mayor facilidad de los hombres para ascender por la escala salarial (Scholarios y Taylor, 2010; Smith, 2008). En la industria los hombres suelen pasar de obrero no cualificado a cualificado y eventualmente a encargado, mientras que en el caso de las trabajadoras la movilidad suele ser más difusa y contradictoria (Daune-Richard, 2005) e incluso inexistente, en un fenómeno que algunos autores han denominado suelo pegajoso (sticky floor) (Booth et al., 2003; Joo Kee, 2006) o peonaje industrial femenino (Babiano, 2007; Fernández Gómez, 2003) para referirse al estancamiento de las trabajadoras en los escalones más bajos de las clasificaciones profesionales.

El menor salario que cobran las mujeres se ha vinculado igualmente con la infravaloración de las tareas que realizan. Una postura en este debate considera que la definición de las cualificaciones no es una operación técnica sino que se constituye a partir de la distinción entre masculino y femenino (Laufer et al., 2005). El trabajo cualificado es masculino y el semi-cualificado o descualificado, femenino (Phillips y Taylor, 1980). Otra postura relacionada sostiene que es necesario analizar en detalle las diferencias que existen entre las tareas masculinas y femeninas, con el fin de detectar los procesos a través de los cuales esas diferencias dan lugar a la desigualdad salarial (Cockburn, 1981; West, 1990).

En la industria, las mujeres tradicionalmente han desarrollado, por un lado, los trabajos más repetitivos y monótonos. Por otro lado, con mucha frecuencia, las tareas de las mujeres se asocian con la destreza o agilidad manual. Esos irreemplazables dedos de hada o little fingers que supuestamente convertían a las mujeres en las candidatas ideales para los trabajos industriales que requerían minuciosidad (Fernández Gómez, 2003; Glucksmann, 1986; Maruani, 1991).

La pregunta que se plantea es por qué la destreza manual o la capacidad de aguantar el trabajo repetitivo no se reconocen salarialmente. Una posible respuesta estriba en su naturalización (Adkins y Lury, 1999; Belt et al., 2002; Walby, 2011). Las aptitudes que las mujeres aportan al trabajo se consideran innatas, vinculadas a la naturaleza femenina y, por tanto, no susceptibles de ser reconocidas monetariamente (Daune-Richard, 2005).

La falta de reconocimiento de las llamadas cualidades naturales femeninas contrasta con la mayor valoración de la que son objeto otras habilidades atribuidas a los hombres como la fuerza física (Kergoat, 2003; Maruani, 1993). Se ha apuntado además que las trabajadoras tienen problemas para que se reconozcan sus supuestas habilidades naturales debido a que no son consideradas "individuos en el trabajo", sino que forman parte del grupo social de las "trabajadoras" (Adkins y Lury, 1999:604). Este proceso, según estas autoras, no se produce en el caso de los hombres, que tienen mayor facilidad para utilizar sus capacidades como un recurso individual con el que negociar.

La discusión sobre la tradicional infravaloración de las tareas femeninas ha puesto de manifiesto el papel que juegan los mecanismos de fijación salarial en el man- 
tenimiento de las desigualdades retributivas (Acker, 1989, 2006). Los sistemas salariales determinan "la visibilidad y la invisibilidad de las competencias" a través del lenguaje universal de la moneda (Daune-Richard, 2005:182).

\section{Problemas con la aplicación del principio de igual retribución por trabajos de igual valor}

La importancia de los sistemas salariales se refleja también en las principales estrategias que se han diseñado para abordar la brecha salarial, y entre las que cabe destacar la regulación del principio de igual salario por trabajo de igual valor (Whitehouse et al., 2001). Este principio busca ir más allá del reconocimiento de la igualdad salarial ante la realización de un mismo trabajo, puesto que con frecuencia hombres y mujeres desempeñan puestos diferentes (Rudin y Byrd, 2003). Se trata de que las mujeres reciban la misma retribución que un varón cuando realicen un puesto de trabajo que pueda ser considerado equivalente, aunque el contenido del mismo sea diferente.

Este principio, reconocido por la OIT en 1951, ha sido adoptado en las regulaciones de numerosos países. El amplio reconocimiento legislativo contrasta, sin embargo, con la reducida efectividad que estas normas han tenido tanto entre los Estados miembros de la Unión Europea (Comisión Europea, 2007; Rubery et al., 2005) como en otros países desarrollados (OIT, 2008). Una de las razones apuntadas ha sido la debilidad del marco normativo. Con frecuencia las leyes sobre la aplicación del principio de igual valor contienen estipulaciones que permiten a las empresas continuar con sus prácticas discriminatorias (Whitehouse et al., 2001). En otros casos, la ley solo se aplica cuando las trabajadoras presentan una demanda, hecho que supone un riesgo que pocas mujeres pueden asumir, y que además suele implicar largos procesos judiciales (Armstrong y Cornish, 1997).

Frente a estas regulaciones, a finales de los años ochenta diferentes provincias canadienses -Manitoba, Prince Edward Island, Nova Scotia, New Brunswick, Ontario-, respondieron a la presión de los sindicatos y de los grupos de mujeres aprobando leyes que obligaban a desarrollar planes de igualdad salarial en las empresas sin necesidad de que se produjeran denuncias previas. Todas estas leyes establecían: sanciones importantes para evitar su incumplimiento; plazos para el desarrollo de los planes de igualdad salarial, con el fin de evitar las estrategias de dilación del pasado; y su aplicación en el sector público (Armstrong y Cornish, 1997). Pero solo la ley de Ontario en 1988 y la de Québec en 1996 afectaron a las empresas privadas. La obligatoriedad de su aplicación, junto con la cobertura de los sectores público y privado, ha llevado a considerar la legislación de estas dos provincias como un modelo que debería ser imitado (OIT, 2008; Peng y Singh, 2009).

La puesta en práctica de estas normas más progresistas se ha topado, no obstante, con algunos obstáculos. En Ontario, la ausencia de mecanismos de seguimiento en la aplicación de la ley, junto con la interpretación que de la misma hicieron algunas compañías, permitió la aprobación de planes que apenas ofrecían mejoras salariales 
a las trabajadoras, sobre todo en las empresas que carecían de presencia sindical (Armstrong y Cornish, 1997). En el sector público, las expectativas depositadas en la legislación de Ontario se vieron reducidas por los sucesivos recortes producidos en los fondos que, inicialmente, se habían previsto para hacer frente a los ajustes salariales motivados por su aplicación (Peng y Singh, 2009).

Además de la falta de voluntad política en la regulación efectiva de la igualdad salarial, se han señalado otros problemas en la aplicación del principio de igual valor. Algunos de ellos hacen referencia a las relaciones entre capital y trabajo, y otros a las relaciones de género. Entre los primeros cabe señalar la oposición de los empleadores al incremento de las retribuciones que su aplicación implica (Acker, 1989; Hart, 2002; Rubery et al., 2005; Whitehouse et al., 2001). Esta resistencia se ha visto reforzada por la tendencia a la disminución de los costes salariales a través de mecanismos como las reestructuraciones, el empleo precario, las privatizaciones o las subcontrataciones (Acker, 2006). Reducir costes se contradice con los objetivos de la igualdad salarial, pues supone disminuir retribuciones no incrementarlas, tal como sucedería si se equipararan los salarios de las trabajadoras a los niveles equivalentes de sus colegas masculinos.

Los factores relacionados con las relaciones de género hacen referencia, por una parte, a la falta de movilización de los agentes sociales en torno a la infravaloración de las tareas femeninas en los dispositivos de clasificación profesional (Rubery et al., 2005; Daune-Richard, 2005). Y de otra, a la oposición masculina a las políticas de igualdad. Reducir las diferencias con los colectivos desfavorecidos puede ser considerado como un ataque a la dignidad y masculinidad (Cockburn, 1991; Hart, 2002).

Una última dificultad en la aplicación del principio de igual valor estriba en que si bien los mecanismos de fijación de retribuciones son un aspecto importante en la lucha contra la brecha salarial, existen otros elementos que también influyen en ese proceso (Wajcman, 2000; Acker, 2006). Este obstáculo no invalida la utilidad del principio de igual valor. Pero recuerda la conveniencia de que, en el ámbito político, las medidas de igualdad aborden los diferentes elementos que dan lugar a la brecha salarial. En la práctica investigadora es necesario también vincular la desigualdad salarial con los diversos aspectos que determinan la diferente posición de hombres $\mathrm{y}$ mujeres en los centros de trabajo (Jones y Torrie, 2009; Whitehouse, 2003).

\section{Metodología}

Dada la variedad de razones que pueden motivar las desigualdades salariales entre sexos se adoptó una metodología cualitativa. A diferencia de la metodología cuantitativa en la que solo se produce la información prevista en el cuestionario, la perspectiva cualitativa permite reflejar los temas que los participantes consideran relevantes con respecto al objeto de investigación.

El diseño de la investigación en la metodología cualitativa tiene también un carácter flexible que posibilita añadir matices o modificaciones según se avanza en el 
proceso investigador (Miles y Huberman, 1994). Nosotras comenzamos realizando doce entrevistas exploratorias (a ocho hombres y cuatros mujeres) en las que obtuvimos información genérica sobre la situación de las mujeres en los principales subsectores de dos comunidades autónomas y datos más concretos sobre cuatro grandes centros de trabajo pertenecientes a actividades en las que abunda la mano de obra masculina (un fábrica de cerveza, una empresa cárnica, una fábrica de productos lácteos y una empresa de bebidas refrescantes) ${ }^{23}$. Con el fin de llegar a los sectores feminizados y recoger la opinión directa de las mujeres, procedimos a realizar ocho grupos de discusión, seis entre 2005 y 2006, y otros dos en 2009. Una razón para recurrir a esta técnica era de tipo económico, en un solo desplazamiento podíamos recoger la opinión de un conjunto de personas. La segunda razón estriba en que los grupos de discusión se consideran una técnica muy pertinente para analizar las relaciones entre sexos porque el género es un proceso interactivo (Frenkel, 2008). Aunque los individuos contribuyen a este proceso, son el intercambio y la negociación entre las aportaciones individuales las que permiten observar cómo el género se construye a través de discursos, unos dominantes y otros alternativos (Hollander, 2002). En este sentido, la dinámica de los grupos de discusión se asemeja a las interacciones que tienen lugar en el contexto social más amplio.

La muestra o selección de participantes también utiliza criterios diferentes en la metodología cuantitativa y cualitativa. En la primera sigue criterios de representatividad estadística, mientras que en la perspectiva cualitativa utiliza criterios como la heterogeneidad o diversidad de los integrantes y la saturación o redundancia estructural de las variables relevantes para el estudio (Vallés, 1999). En el diseño de los

${ }^{2}$ La industria de alimentación y bebidas (códigos 10 y 11 en la CNAE 2009) incluye sub-sectores en los que a nivel agregado -y según los datos de la EPA - el peso del empleo femenino es minoritario: fabricación de productos lácteos, donde las mujeres constituyen el $34 \%$ de la población asalariada, la fabricación de bebidas ( $26 \%$ de asalariadas) y la elaboración de productos cárnicos (29\% de mujeres). Otros sub-sectores dentro de esta actividad se caracterizan por una proporción mayoritaria de asalariadas. Este es el caso del procesado y conservación de pescados, donde las asalariadas representan el 65\% del empleo, así como del procesado y conservación de frutas y hortalizas (52\% de mujeres).

${ }^{3}$ Se entrevistó a un responsable de la federación agroalimentaria de CC.OO. en Cantabria y a otro de Murcia. El primero proporcionó información sobre la industria láctea, las conservas de pescado y el sector cárnico en esa comunidad autónoma. Y el segundo, sobre el manipulado de frutas y hortalizas. Se llevaron a cabo tres entrevistas entre representantes sindicales de CC.OO. - dos hombres y una mujer - en tres grandes empresas dedicadas a la fabricación de productos lácteos (Cantabria), productos cárnicos (Burgos) y bebidas no alcohólicas (Madrid). Cuatro de estas cinco entrevistas se realizaron por teléfono. Las siete entrevistas restantes formaron parte de la visita que hicimos a las instalaciones de una gran fábrica cervecera en Madrid. En esa fábrica, nos reunimos con cuatro representantes sindicales varones de CC.OO. y entrevistamos a tres trabajadoras de distintos departamentos (producción, laboratorio y envasado). 
grupos de discusión se han tenido en cuenta las siguientes variables: ubicación geográfica (con cinco comunidades autónomas cubiertas); peso de la mano de obra femenina en el empleo ${ }^{4}$ y sexo de los asistentes (cinco grupos eran mixtos, dos estaban compuestos solo por mujeres, y uno por hombres). En el anexo aparecen las principales características de los grupos.

En las entrevistas y en los grupos se utilizó de manera flexible un guión de temas que se fue mejorando según avanzaba el trabajo de campo, tal como es habitual en los estudios cualitativos (Vallés, 1999:203). El guión contenía los siguientes temas: características de la negociación colectiva y de las empresas; peso y características del empleo femenino; categorías femeninas y masculinas; tareas desempeñadas; demandas sobre clasificación profesional; movilidad ocupacional; conciliación; y perspectivas de cambio. No obstante, en los grupos de discusión se intentó que la moderación fuera lo menos dirigida posible con el fin de evitar, en línea con lo defendido por Callejo (2005), que los participantes respondieran a una lista de temas preestablecidos por la investigadora. El tema de discusión que se introdujo en cada grupo fue la situación de las mujeres en cada sector y a continuación se pedía a cada asistente que se presentara y explicara las características de su empresa en relación al problema planteado. En general, el propio grupo de discusión cubría de forma espontánea los temas reflejados en el guión, excepto los permisos asociados con la conciliación que solían ser introducidos por la moderadora al final de la discusión.

Los integrantes de los grupos de discusión eran en su mayoría representantes sindicales de CC.OO. en sus empresas y ello por diferentes motivos. El primero se refiere a la accesibilidad, era más fácil llegar a ellos porque contábamos con la ayuda de las federaciones agroalimentarias del sindicato a la hora de contactar y seleccionar a las personas participantes. La segunda razón estriba en que, por su papel de representantes del resto de los trabajadores, suelen tener una visión de conjunto, sobre las principales demandas en su centro de trabajo y las posibilidades de cambio, de la que carece el personal sin estas responsabilidades. En este sentido, se intentó que los integrantes de los grupos de discusión fueran delegados de base, es decir, que no pertenecieran a las estructuras sindicales responsables de elaborar las estrategias sindicales. Tratábamos de reflejar, no tanto el discurso sobre los objetivos que se perseguían, sino la experiencia diaria en los centros de trabajo. El resultado final fue que de 53 personas participantes en los grupos (40 mujeres y 13

${ }^{4}$ En los grupos de discusión se cubrieron sub-sectores de la industria alimentaria masculinizados, como la industria láctea, y otros feminizados, como el procesado y conservación de pescados, así como de frutas y hortalizas. Una idea que interesa resaltar es que encontramos una minoría de empresas cuya composición del empleo por sexos no coincide con los datos agregados que presenta la EPA. Por ejemplo, se celebró un grupo en una fábrica de galletas donde las mujeres representan el $75 \%$ de la plantilla, cuando se trata de un subsector, la fabricación de panadería y pastas alimenticias, en el que las mujeres a nivel agregado suponen el 44\% del empleo. 
hombres), solo tres pertenecían a la estructura sindical del sector (un representante estatal en el grupo 2, una representante comarcal en el grupo 3 y otra provincial en el grupo 8).

Las reuniones de grupo se grabaron y transcribieron, y en las entrevistas se tomaron notas. En el proceso de análisis e interpretación se ha buscado identificar las prácticas que contribuyen a mantener a las mujeres en una situación de subordinación, así como los discursos y representaciones sobre las mismas. Para ello se han seguido varias fases, similares a las propuestas por Callejo (2001). Una primera en la que se ha llevado a cabo un análisis por temas de cada grupo de discusión. En esta fase hemos buscado la posición o posiciones del grupo con respectos a los siguiente temas: exclusión de las mujeres/privilegios masculinos; infravaloración de las tareas femeninas (categorías y salario); condiciones de trabajo de las mujeres (ritmos de trabajo, impacto en la salud); movilidad ocupacional; y perspectivas de cambio. En la segunda fase, se ha comparado el análisis de todos los grupos entre sí con el fin de detectar las similitudes y diferencias. La dificultad para atribuir afirmaciones de los grupos a criterios de género se ha resuelto mediante la recurrencia (Ibíd., 156): la misma afirmación en más de un grupo de discusión realizada por personas del mismo sexo confirma el género como condicionante ${ }^{5}$. En la última fase, hemos revisado nuevamente las transcripciones de los grupos intentando refutar los resultados de las fases anteriores.

Nuestro análisis se centra en las trabajadoras y trabajadores pertenecientes al denominado grupo obrero, que es el colectivo más numeroso y donde mayor representatividad tienen los sindicatos. No se abordan los problemas de desigualdad salarial que se pueden producir en grupos ocupacionales como los mandos intermedios, el personal técnico, las administrativas o los directivos.

\section{Resultados}

Las obreras en la industria agroalimentaria tienen un estatus laboral inferior a sus compañeros. Esto se observa en diferentes procesos que influyen en los salarios que perciben. A continuación analizamos estos procesos y los mecanismos que se utilizan para justificarlos. Finaliza esta sección con un apartado sobre las posibilidades de cambio.

\subsection{La exclusión de las mujeres de los mejores empleos y el peso de los estereotipos}

Los empleos mejor remunerados en las fábricas de la industria agroalimentaria suelen estar reservados a los hombres. En este sentido, cabe señalar dos tendencias en la composición de las plantillas que se repiten con frecuencia. Por un lado,

\footnotetext{
${ }^{5}$ A lo largo del artículo se incluye entre paréntesis el número de grupo de discusión y la página de la transcripción en la que aparecen las citas que apoyan las diferentes afirmaciones.
} 
existen fábricas con niveles salariales altos que no contratan trabajadoras en los departamentos que mayor volumen de empleo generan, y que suelen ser los de envasado y producción. Este es el caso de empresas pertenecientes a sectores en los que a nivel agregado abunda la mano de obra masculina, como la industria láctea $o$ de bebidas. Por otro lado, encontramos centros de trabajo con una composición mixta del empleo, pero en las que suele haber una fuerte segregación entre los puestos de trabajo masculinos y femeninos. El problema de estos centros estriba en que esta división sexual del trabajo se acompaña de una serie de ventajas salariales para los hombres, a las que difícilmente acceden las mujeres. Se trata de una situación habitual en los sectores en los que a nivel agregado la mano de obra femenina es mayoritaria, tales como la elaboración y conservas de pescado o la preparación y conservación de frutas y hortalizas (sectores a los que por brevedad denominaremos conservas de pescado y conservas vegetales, respectivamente).

Otra muestra de cómo las situaciones que implican mejores perspectivas salariales se reservan a los hombres, consiste en la mayor facilidad que las empresas conceden a los varones para realizar y cobrar las horas realizadas por encima de la jornada habitual (GD1, GD6:26). Las horas extras se utilizan además para garantizar a los hombres unos ingresos regulares, independientemente de la carga de trabajo disponible (GD3:9).

¿Por qué las empresas dispensan ese trato preferente a los varones? Esta era una cuestión que se planteaban con frecuencia las personas integrantes de los grupos de discusión. Diversos factores se utilizan para explicar las ventajas salariales de los hombres, tales como el salario familiar masculino, la relación de las mujeres con la tecnología o la mayor fuerza física de los hombres.

Los comentarios sobre el salario familiar masculino (breadwinner) aluden a dos de los usos de este concepto (Warren, 2007): para referirse al hombre como el principal proveedor financiero de la familia y como una ideología que influye en la identidad masculina y en sus expectativas.

En el grupo de discusión de la industria láctea, se buscan razones para explicar por qué la fábrica de quesos en la que trabaja una de las integrantes del grupo emplea a un $90 \%$ de mujeres, cuando se trata de un sector en el que abunda la mano de obra masculina. Los integrantes varones de dicho grupo consideran que se debe a los bajos salarios que se pagan.

Lo interesante de esta discusión es que pone de manifiesto la pervivencia de la idea del salario familiar masculino y el papel secundario del salario femenino. Según los hombres, la mujer estaría dispuesta a soportar salarios bajos, porque sus retribuciones se consideran accesorias en la economía familiar, pero al varón le resultaría más difícil al tener que mantener una familia (GD7:10). Ante esto, los varones buscarían otros empleos mejor remunerados. Esa búsqueda se vincula con la identidad masculina en el interior de la pareja. Se antepone el pasado en el que los salarios masculinos siempre eran superiores con la amenaza actual de que la mujer pueda ganar más que su pareja. Esto llevaría a los hombres a acentuar su búsqueda de trabajos mejor remunerados. 
Esa mentalidad un poco machista pues no, no te deja, porque yo conozco mucha gente que ¿cómo va a trabajar la mujer y yo gano menos?, no les cabe en la cabeza, (hombre, GD7:13-14).

La cuestión es hasta qué punto el modelo de salario familiar masculino determina también la retribución de las mujeres y los puestos a los que se les permite acceder. De tal forma, que los empleos con mejores perspectivas salariales o el pago regular de horas extraordinarias se consideran patrimonio de los varones porque todavía pervive la idea de que el hombre ha de ganar más en el interior de la pareja. Se trata de una afirmación que alguna mujer verbaliza (GD3:23), pero que en la mayoría de las ocasiones permanece implícita. En este sentido, constituye un buen ejemplo del diálogo que se produce en las discusiones de grupo con un "interlocutor no visible", cuyas palabras no se oyen pero determinan el discurso de los otros interlocutores (Callejo, 2001: 54).

Otro aspecto relevante estriba en cómo se aplica el modelo del salario familiar masculino con el incremento de los hogares en los que no existe un varón, como es el caso de las familias dirigidas por mujeres (Warren, 2007). Se da la paradoja de que el supuesto salario familiar no se hace extensivo a las mujeres que sustentan un hogar pero sí a los hombres que carecen de esas responsabilidades.

Además del salario familiar masculino, un segundo factor que utilizan los varones se refiere a los estereotipos sobre la relación de las mujeres con la tecnología. Varios elementos se entremezclan en el discurso masculino para explicar la ausencia de las mujeres de determinadas situaciones asociadas con el uso de la tecnología, tales como el departamento de mantenimiento así como puestos relacionados con la introducción de nueva maquinaria o que requieren intervenir en las máquinas.

Se recrimina a los empresarios por asumir que los hombres tienen mayores habilidades para manipular la maquinaria (GD2:9, GD3:7, GD7:15). Se culpa a las mujeres (y se disculpa a los empresarios) por no adquirir formación en las especialidades que supuestamente dan acceso a esos empleos. Este discurso asume que como las mujeres tienen la oportunidad de formarse en mecánica, electrónica o electricidad, su ausencia de los puestos relacionados con la tecnología constituye un problema individual y no social (Flecker et al., 1998). Una dificultad con este argumento estriba en que cuando se indaga en si realmente a los hombres se les exige dicha titulación, se descubre que con frecuencia las empresas forman a sus propios trabajadores (GD1, GD7:17, GD4:5).

Se reprocha también a las mujeres su incompetencia tecnológica. Se trata de explicaciones que, en los grupos de discusión mixtos, los hombres rápidamente matizan pues son conscientes de que estamos ante una construcción social (GD3:17, GD7:18). Las mujeres rechazan los estereotipos sobre su relación con la tecnología. Ellas conocen bien las máquinas en las que trabajan, son capaces de detectar las averías a través de cambios en el ruido de los engranajes (GD7:18, GD3:21-22). No realizan tareas de manipulación y mantenimiento de las mismas porque no se lo permiten (GD3:19); o porque son funciones que no van a ser reconocidas salarialmente sino que solo van a servir para aliviar la carga de trabajo de los varones 
(GD3:23). Este último elemento pone de manifiesto lo enraizada que está la división sexual del trabajo y la dificultad de las mujeres para conseguir acceder a las ventajas salariales de los varones. Los hombres también reconocen estas dificultades cuando explican cómo los mandos intermedios cubren las vacantes que exigen intervenir en la maquinaria con varones, en lugar de asignárselas a las mujeres que conocen el funcionamiento de esas máquinas. La razón que ofrecen consiste en que son trabajos que, por su estatus, se consideran reservados a los varones (GD3:17).

La fuerza física es, junto al salario familiar masculino y la tecnología, otro de los factores que se utiliza como justificación de la exclusión de las mujeres. La dirección de la fábrica de cerveza recurre a la fuerza física como excusa para no contratar mujeres. También los varones de conservas vegetales justifican su patrimonio sobre los puestos que tienen garantizado trabajo durante todo el año aduciendo que son tareas que exigen fuerza física (GD2). Las mujeres, por su parte, hacen referencia a un fenómeno más amplio que cuestiona la vinculación entre fuerza física y masculinidad. En unos entornos de trabajo en los que la distinción entre masculino y femenino condiciona las ventajas y desventajas (Acker, 1990), no es raro que las funciones masculinas de menor estatus, como la manipulación de cargas, se asignen al colectivo más desfavorecido. Las trabajadoras de conservas de pescado señalan que en el trabajo diario es frecuente que las empresas les adjudiquen -y los hombres se desentiendan de- la manipulación de las cargas más pesadas, como puede ser descargar camiones o levantar atunes (GD6:29). El siguiente comentario de una joven canaria condensa bien este fenómeno: "Hombres para los trabajos buenos, para los malos las mujeres somos medio hombres también (GD5:36)".

\subsection{La infravaloración del trabajo de las mujeres}

El menor valor adjudicado a las tareas que desarrollan las mujeres es una realidad en el sector agroalimentario. Este fenómeno se produce independientemente de las funciones que realicen las obreras, puesto que sucede no solo cuando las mujeres llevan a cabo los trabajos que tradicionalmente se le han asignado en la industria, sino también cuando desarrollan otras tareas. Dos elementos interrelacionados influyen en ese proceso: la división sexual del trabajo y los sistemas de clasificación profesional.

La división sexual del trabajo en actividades como la fabricación de galletas sigue las pautas encontradas en otros estudios comparativos (Flecker et al., 1998). El procesamiento de los materiales (amasado) y el mantenimiento son tareas masculinas, mientras que el empaquetado es una labor femenina. Las trabajadoras además están sometidas a un alto grado de polivalencia al rotar por los diferentes puestos de envasado, que incluyen tareas tan variadas como el manejo de maquinaria, apilar cajas, revisar la calidad del producto o colocar el surtido en las cajas. Esta flexibilidad no se reconoce como un elemento cualificante de su trabajo en el sistema de encuadramiento profesional.

Las tareas de los hombres, por el contrario, son objeto de un mayor reconocimiento de su especialización, al ser clasificados en esta fábrica en diferentes grupos de personal (producción, oficios varios, mantenimiento). Pero además esos diferen- 
tes grupos masculinos tienen todos ellos reconocidos los mismos niveles salariales y determinados pluses que no cobran las trabajadoras. Las mujeres son siempre clasificadas en el grupo de personal de envasado. El salario base máximo del grupo de envasado, al que accede una operaria con dificultad después de una década, es equiparable al salario de entrada de un varón. La existencia de escalas salariales diferentes para cada sexo, en las que el salario máximo de las mujeres es idéntico al salario más bajo de los hombres constituye un mecanismo de discriminación salarial clásico (Acker, 1989:6).

Otras situaciones similares las encontramos en el sector de conservas de pescado donde las mujeres son contratadas en el grupo de fabricación y los hombres en el grupo de oficios varios. La categoría de entrada del grupo masculinizado tenía hasta el año 2006, en que se empezó a corregir la situación, un salario superior a la categoría de entrada del grupo feminizado y por encima de las retribuciones del siguiente escalón en el grupo femenino, la oficialía $2^{\mathrm{a}}$. En una fábrica de productos lácteos, las mujeres son clasificadas en los niveles salariales no cualificados, y los hombres en el nivel cualificado. En Murcia, en el convenio de manipulado y envasado de agrios los salarios de las categorías masculinas (peón y cargador) son superiores a los de la categoría femenina (encajadora).

El análisis de las tareas realizadas por cada sexo confirma que las mujeres realizan los trabajos más repetitivos y monótonos, pero también los que requieren minuciosidad y destreza manual. Los hombres y mujeres integrantes de los grupos constatan que los empresarios prefieren asignar estas tareas a las mujeres y se preguntan si se debe a una aptitud innata femenina o a mecanismos sociales. Los hombres en sus respuestas apuntan que la resistencia masculina a realizar estas funciones se explica por el segundo motivo -"cultura machista" (GD3:3), "si no queda más remedio, al final te lo tienes que comer y aguantar" (GD2:20)-.

La pregunta de por qué esas aptitudes no son reconocidas salarialmente no tiene una respuesta fácil. Por un lado, está el peso de tradición, son trabajos que históricamente en las clasificaciones profesionales se han definido como descualificados y femeninos. Pero, por otro lado, las mujeres realizan otras tareas en las fábricas que tampoco se reconocen. Se ha hecho referencia al principio de este apartado a la diversidad de funciones que llevan a cabo en la fábrica de galletas. Además los trabajos de las mujeres implican con frecuencia la realización de esfuerzos físicos importantes (GD1, GD3, GD5, GD6, GD7, GD8). Y, en ocasiones, como se ha mencionado en el epígrafe 5.1, se le asignan unas tareas que en la industria se suelen asociar con el trabajo masculino, la manipulación de cargas muy pesadas.

En su intento por explicar la falta de reconocimiento del trabajo que desarrollan las mujeres, algunas autoras apuntan que el mundo del trabajo y de la empresa está estructurado por un orden de los sexos por el cual lo masculino domina material y simbólicamente (Daune-Richard, 2005). La jerarquía social de lo masculino y lo femenino hace que las habilidades ejercidas por las trabajadoras permanezcan invisibles en las escalas de evaluación del trabajo, incluso cuando las mujeres utilizan las mismas cualidades que se retribuyen en los varones. La pregunta es 
entonces si esta jerarquía de los sexos se impone sin más a los actores, determinando su comportamiento.

En nuestra opinión, es necesario indagar en los mecanismos que los distintos actores ponen en práctica para mantener el statu quo. Así, las empresas recurren a una estrategia de grupalidad hacia las mujeres en su intento por mantener los salarios bajos. Una herramienta útil para ello es utilizar los grupos de clasificación como compartimentos estancos, sin permeabilidad entre las categorías masculinas y femeninas. Cabe señalar que para este propósito resulta útil mantener en los convenios las antiguas clasificaciones profesionales de las ordenanzas laborales con su diferenciación entre categorías y grupos masculinos y femeninos. Este mecanismo además acentúa la división sexual del trabajo. Si las mujeres desarrollan puestos asociados con los hombres, cabe el riesgo de que las reclamaciones salariales de las trabajadoras puedan servir para romper con esa estrategia de grupalidad. Un ejemplo de esta política empresarial se encuentra en la fábrica de galletas, donde se utiliza el grupo de envasado con sus menores salarios para clasificar a todas las mujeres, incluso cuando desarrollan tareas difícilmente encuadrables en ese grupo, como es el caso del manejo de la carretilla o las funciones de laboratorio.

\subsection{El mayor control al que están sometidas las mujeres}

Se ha sugerido que las mujeres sufren modalidades de control más rígidas que los hombres (Beechey, 1994; Kergoat, 2003), pero este fenómeno no se ha relacionado directamente con la desigualdad salarial. En nuestros resultados este mayor control se produce en tres dimensiones: las funciones que se asignan a las mujeres, las relaciones de autoridad y la promoción ${ }^{6}$.

Una primera forma de control se aprecia a través de las tareas adjudicadas a las trabajadoras, sobre todo en los sectores feminizados, pero no solamente puesto que también se da en algunas grandes empresas de productos lácteos. Se trata de tareas que requieren durante buena parte de la jornada laboral destreza y atención permanente, mantener posturas incómodas o sedentarias, realizar movimientos repetitivos, o trabajar al ritmo que marca la cinta de producción o el cronómetro. Los varones de conservas vegetales consideran que estas funciones constituyen los "peores trabajos" (GD2). Las tareas de los hombres se caracterizan, en general, por una mayor diversidad y comodidad. Levantar objetos pesados representa la parte más incómoda del trabajo masculino, tarea ésta que los propios hombres consideran menos "sacrificada" que el trabajo femenino (GD2:19-20). Las trabajadoras perciben el mismo fenómeno de otra manera. En actividades tan diferentes como la recolección y cultivo de flores y las conservas de pescado, y en puntos geográficos tan distantes entre sí como Canarias, Galicia o Cantabria, las mujeres coinciden en

${ }^{6}$ Estas tres dimensiones son similares a las propuestas por Littler $(1982,1990)$ para el análisis de las estrategias utilizadas por las empresas en la organización del trabajo. 
afirmar que la carga y el ritmo de trabajo de los varones son muy inferiores a las suyas. Afirman literalmente "los hombres no hacen nada" (GD5:34, GD6:3).

La segunda forma de control es la ejercida a través de las estructuras de autoridad de las fábricas. En este caso destacan las interacciones informales que tienen lugar en el desarrollo diario del trabajo (Acker, 2006). Se trata de prácticas que dan lugar a desigualdades sutiles que solo denuncian las mujeres. Así, las normas disciplinarias son, en ocasiones, diferentes para cada sexo. Los mandos intermedios ejercen un mayor control sobre el ritmo de trabajo de las trabajadoras, mientras los varones disfrutan de más autonomía en el desarrollo de sus tareas (GD3:11, GD8:2). Prohibiciones como trabajar sin gorro (GD6) o con pendientes (GD5) se aplican solo a las mujeres. Otras veces se utilizan distintas reglas para medir el trabajo femenino y masculino. En una empresa agrícola canaria los varones terminan su jornada laboral entre una y dos horas antes, mientras que las mujeres han de realizar la jornada completa (GD5). En este caso, el trabajo de las mujeres se mide en tiempo, que no se ha de malgastar, mientras que el de los hombres en tareas; cuando se acaba la tarea, termina su jornada ${ }^{7}$.

La tercera forma de control se concreta en el peonaje industrial femenino. Si bien ambos sexos están sometidos a la arbitrariedad de las empresas en la promoción, se observa un patrón común en los sectores feminizados por el que los hombres ascienden a las categorías superiores con mayor rapidez que las mujeres. El ascenso para las trabajadoras suele estar sometido a obstáculos y reglas que no se vinculan con las tareas realizadas, sino con la consideración de grupo desfavorecido al que solo se le permite la promoción cuando son capaces de organizarse de forma colectiva y presionar para conseguirlo. En la categoría de oficial de $1^{\text {a }}$ es donde mejor se observa el rechazo de las empresas a reconocer una progresión salarial a las mujeres. No es una categoría habitual entre las trabajadoras. Ante este hecho, las representantes sindicales en el sector de conservas de pescado intentan vincular la oficialía $1^{\mathrm{a}}$ a la edad de jubilación, de forma que las empresas reconozcan esa categoría solo a las mujeres que están próximas a jubilarse, con el fin de que vean elevadas sus bases de cotización a la Seguridad Social, aspecto éste que luego influye en la pensión que se recibe (GD8:28).

La excepción a este patrón de falta de movilidad ocupacional en los sectores feminizados la encontramos en el denominado envasado de cuarta gama, dentro del sector de conservas vegetales (GD4). Se trata de empresas de manipulado y envasado de productos como ensaladas frescas que cuentan con convenio propio, lo que implica mayores salarios, y con una plantilla más joven que las empresas representadas en el grupo de discusión 3, pertenecientes también al sector de conservas vegetales, pero cubiertas por el convenio estatal. Aunque la mano de obra femenina

${ }^{7}$ Thompson (1967/1979: 247) utiliza esta diferenciación entre tarea y tiempo de trabajo para contraponer la menor autonomía del trabajador asalariado frente al campesino independiente. 
es mayoritaria en el envasado de cuarta gama, una parte de las asistentes al grupo de discusión no se identifica con los patrones de desigualdad observados en el resto de sectores feminizados. La diferencia se encuentra precisamente en que en estas empresas las mujeres también tienen derecho a la movilidad ocupacional ascendente. Existe además en estos centros de trabajo una cultura empresarial desprovista del sexismo y autoritarismo que se observa en los demás sectores feminizados.

Además del ascenso a las oficialías, otra vía de movilidad ocupacional interna consiste en el acceso a los empleos con mayor retribución y mejores perspectivas de promoción. Las mujeres encuentran también obstáculos para acceder a estos puestos. Dos ejemplos que se repiten en distintos grupos de discusión son el manejo de la carretilla y los puestos de mantenimiento. En el primer caso, empieza a ser habitual encontrar mujeres conduciendo carretillas en las fábricas, pero no siempre consiguen que se les retribuya igual que a los hombres ni que se les reconozca la mima categoría (GD1, GD3:5). En la siguiente cita se puede apreciar la excepcionalidad que supone reconocer esta categoría a una trabajadora: "había solamente una carretillera [...] y estaba con su categoría y todo" (hombre, GD2:5). Las mujeres, sin embargo, no han conseguido entrar en los departamentos de mantenimiento. El único caso que se menciona en el trabajo de campo es el de una empresa en la que los trabajadores de ese departamento recurrieron a distintas estrategias de exclusión hasta conseguir que se fuera la única operaria que había conseguido acceder a un puesto de mantenimiento (GD4:26).

¿Cómo influyen las tres dimensiones señaladas en los salarios? En relación a la movilidad ocupacional, está claro que a menor categoría menor salario. En cuanto a las tareas realizadas y las relaciones de autoridad, el efecto salarial más evidente consiste en la pérdida de ingresos que conlleva el deterioro de la salud. En todas las empresas feminizadas son frecuentes las lesiones en las extremidades superiores, inferiores y en la espalda. Estas lesiones disminuyen las retribuciones actuales y diferidas de las mujeres. Por un lado, durante las situaciones de incapacidad temporal la retribución es inferior a la que se obtiene cuando se está trabajando. Por otro, es común que, debido al desgaste físico que padecen, las mujeres abandonen la actividad laboral antes de jubilarse, bien con despidos pactados, bajas voluntarias, prejubilaciones o por reconocimiento de una incapacidad laboral.

\subsection{Perspectivas de cambio}

Superar la infravaloración de las tareas femeninas constituye la estrategia de cambio sobre la que más se ha intentado actuar. Incrementar la presencia de las mujeres en los sectores y puestos mejor remunerados no es un objetivo que se esté debatiendo. En cuanto al mayor control que padecen las mujeres, sindicalmente se defienden algunas medidas dirigidas a aliviar las repercusiones en la salud y el salario, tales como la rotación entre puestos o adelantar la edad de jubilación de las mujeres en el sector de conservas de pescado.

En relación a la equiparación salarial entre sexos, se ha conseguido igualar los salarios entre las categorías femeninas y masculinas en los convenios estatales de dos sectores feminizados, las conservas vegetales y las conservas de pescado. En 
ambos casos, se han equiparado los salarios de la categoría más baja de cada sexo. Además, en el segundo caso, se ha reducido, aunque no totalmente, la diferencia entre las oficialías segundas de ambos sexos, pero se ha mantenido la distancia salarial existente entre las oficialías de primera de las categorías masculinas y femeninas.

Cuando no ha sido posible conseguir la equiparación salarial a través de la negociación colectiva, la otra vía seguida ha sido el recurso a los tribunales. En Murcia, los sindicatos denunciaron el convenio provincial de manipulado y envasado de agrios por contemplar para las mujeres salarios inferiores a los masculinos, tal como se ha señalado en el epígrafe 5.2. Los tribunales dieron la razón a las empresas, que justificaron los mayores salarios masculinos en el uso de la fuerza física.

Las dificultades para demostrar la equivalencia de las categorías femeninas y masculinas del ejemplo anterior apunta a un aspecto clave de estos procedimientos: cómo determinar el valor de los puestos de trabajo. La convención número 100 de la OIT sobre igualdad de remuneración recomienda "adoptar medidas para promover la evaluación objetiva del empleo". En este sentido, se considera que los sistemas de valoración de puestos de trabajo -también denominados sistemas analíticos - pueden ser más objetivos que los sistemas de clasificación (Armstrong et al., 2007; Comisión Europea, 1994; Parlamento Europeo, 2002). Los primeros se basan en la descomposición de cada puesto en sus principales componentes y la asignación de puntos a cada componente; mientras que en los segundos el valor de cada puesto se estima de forma global, lo que puede dar lugar a la introducción de criterios de valoración sesgados. Sin embargo, los sistemas analíticos no son inmunes a los sesgos sexistas a no ser que se diseñen explícitamente para abordar el principio de igual valor (Armstrong et al., 2007).

En España, a diferencia de otros países como el Reino Unido donde los sistemas de valoración de puestos son un instrumento habitual para el logro de la equiparación salarial (Whitehouse et al., 2001), no existe esta tradición ni la legislación establece nada al respecto. En un análisis de 155 convenios colectivos (39 de ámbito sectorial estatal y 116 de empresa) tan solo trece convenios de empresa aluden al uso de este sistema (Morillo, 2006). Se trata de empresas de capital extranjero en su mayoría, seis de ellas en el sector del automóvil, y que no justifican el uso de la valoración de puestos como forma de aplicar el principio de igual valor.

Un ejemplo de cómo los sistemas analíticos por sí solos no garantizan la equidad salarial se encuentra en el proceso de negociación de un nuevo sistema de clasificación profesional en la fábrica de galletas. Este proceso negociador se inicia tras celebrar las mujeres una huelga en la que demandaban medidas para acabar con la infravaloración de las tareas femeninas ${ }^{8}$. La empresa logró varios objetivos al recurrir a la valoración de puestos de trabajo, ninguno de los cuales implicaba

${ }^{8}$ Tanto la huelga como la negociación de la nueva clasificación profesional son posteriores a la celebración del grupo de discusión en esa fábrica. 
aplicar el principio de trabajo de igual valor ${ }^{9}$. En primer lugar, intentaba evitar el proceso de negociación al contratar a consultores externos que no informaban a la parte sindical de uno de los posibles sesgos sexistas de estos sistemas, la puntuación y el peso asignado a cada componente (Armstrong et al., 2007). La puntuación de los distintos componentes se suma para cada puesto de trabajo con el fin de elaborar un ranking de puestos que luego se utiliza como justificación para adjudicar retribuciones. En segundo lugar, distinguir múltiples puestos en lo que hasta entonces ha sido un único grupo profesional con tres categorías (auxiliar, oficial $2^{\mathrm{a}}$ y oficial $1^{\mathrm{a}}$ ) permite justificar subidas solo para aquellos puestos que obtienen mayor puntuación, y mantener el resto de los salarios femeninos intactos. Esta estrategia contrasta con la demanda sindical de equiparar los salarios femeninos y masculinos para cada una de las principales categorías (auxiliar, oficial $2^{\mathrm{a}}$ y oficial $1^{\mathrm{a}}$ ), mecanismo que implica un incremento salarial para todo el colectivo femenino. Por último, al ser la valoración de puestos un proceso largo (Whitehouse, 2003), la empresa aprovechó para prolongar la negociación en su intento por evitar el reconocimiento de mayores salarios.

Este último problema remite a otro obstáculo que aparece en la aplicación del principio de igual salario por trabajo de igual valor, la debilidad del marco normativo español (Quintanilla, 1996; Saldaña, 2004). La legislación española no establece plazos para su puesta en práctica ni sanciones por su incumplimiento. En la fábrica de galletas, llevan desde 2007 negociando el nuevo sistema. Solo en la fase de descripción de los puestos se emplearon más de dos años de reuniones entre las partes. Cuando la empresa fue comprada por otros propietarios, la dirección aprovechó para iniciar nuevamente el proceso con un consultor diferente.

Además de las barreras señaladas, en la negociación del nuevo sistema de clasificación profesional en la fábrica de galletas aparece la resistencia masculina. Los hombres, ante la presión de las trabajadoras, aceptan la equiparación salarial en las categorías más bajas pero les cuesta defender esa igualación en las más altas, la oficialía primera en este caso. Tal como han indicado otros estudios de procesos de negociación similares, los varones se resisten a reconocer que el trabajo de las mujeres está tan cualificado como el suyo (Acker, 1989). Su identidad masculina parece residir en mantener la ventaja salarial sobre las trabajadoras (Hart, 2002).

${ }^{9}$ La información sobre esta experiencia se ha obtenido mediante la participación directa de la autora en esa negociación, como asesora de los representantes de CC.OO. entre 2007 y 2010. Como tal, se entrevistó con los trabajadores y trabajadoras con el fin de realizar una descripción de los puestos de trabajo, consultó diversa documentación sobre la empresa y acudió durante esos tres años a las reuniones que se celebraban mensualmente entre la dirección y el comité de empresa. 


\section{Conclusiones}

En este artículo hemos abordado distintos procesos que ayudan a explicar la desigualdad salarial en la industria agroalimentaria. Aunque existe variabilidad entre organizaciones, se han detectado una serie de prácticas que se repiten en diferentes empresas y sectores, tales como la exclusión de las trabajadoras de las situaciones laborales que implican mayores salarios, la infravaloración de los trabajos desarrollados por las mujeres - no solo de las tareas tradicionalmente consideradas femeninas en la industria-, la asignación a las trabajadoras de las tareas más arduas y de menor estatus o el peonaje industrial femenino. Un elemento común a estas prácticas es la consideración de las mujeres como un grupo subordinado al que se le impide acceder a una serie de ventajas que se dan por sentado en el caso de sus homólogos varones. Se constata, por tanto, nuestra hipótesis de que la brecha salarial constituye la condensación de los distintos tipos de desigualdades que sufren las mujeres en el ámbito laboral.

Las expectativas de transformación no son muy halagüeñas. En nuestro país no existe debate sobre los problemas que presenta la actual regulación del principio de igual valor. En la Unión Europea se constatan las deficiencias del modelo actual, pero no se aprecia voluntad política para cambiarlo. La Comisión Europea reconoce que la legislación comunitaria no ha resultado "eficaz para garantizar el respeto del principio de una retribución igual para un trabajo de igual valor" (Comisión Europea, 2007:6), pero considera innecesario modificarla. Esta instancia política se muestra partidaria de seguir manteniendo el mismo enfoque que tan exiguos resultados ha proporcionado, la difusión de buenas prácticas (Comisión Europea, 2009). Dicho enfoque se enmarca además dentro de la pérdida de peso que, en la última década, ha sufrido la igualdad de género en las políticas de empleo comunitarias (Mósesdóttir, 2011), por lo que no parece que en los próximos años el ámbito europeo vaya a liderar reformas progresistas en esta materia.

Otra complicación estriba en la necesidad de que las políticas públicas incidan no solo en la infravaloración de las tareas femeninas, tal como hace el principio de igual valor, sino también en los demás factores que determinan la brecha salarial. Aumentar la presencia femenina en los puestos y sectores mejor retribuidos constituye una medida que contribuiría a mitigar la estrategia de grupalidad y subordinación que utilizan las empresas en su gestión de la mano de obra femenina. Igualmente, parece conveniente seguir indagando en el papel que juegan en las políticas empresariales y las identidades masculinas estereotipos como el salario familiar masculino o su supuesta mayor competencia técnica. 


\section{Anexo}

\begin{tabular}{|c|c|c|c|}
\hline \multicolumn{4}{|c|}{ Características de los grupos de discusión } \\
\hline $\begin{array}{l}\mathrm{N}^{\circ} \text { de } \\
\text { grupo }\end{array}$ & Integrantes & Actividad de las empresas & $\begin{array}{l}\text { Lugar de celebra- } \\
\text { ción }\end{array}$ \\
\hline GD1 & $\begin{array}{l}\text { Cinco mujeres y } \\
\text { un hombre }\end{array}$ & $\begin{array}{l}\text { Empresa dedicada a la fabrica- } \\
\text { ción de galletas con } 75 \% \text { de } \\
\text { mano de obra femenina. }\end{array}$ & $\begin{array}{l}\text { Comunidad de } \\
\text { Madrid }\end{array}$ \\
\hline GD2 & Cuatro hombres & $\begin{array}{l}\text { Conservas de frutas y hortali- } \\
\text { zas. Empresas situadas en } \\
\text { Valencia, La Rioja y Navarra. }\end{array}$ & Madrid \\
\hline GD3 & $\begin{array}{l}\text { Seis mujeres y } \\
\text { dos hombres }\end{array}$ & $\begin{array}{l}\text { Conservas de frutas y hortali- } \\
\text { zas. Empresas sujetas al con- } \\
\text { venio estatal. }\end{array}$ & Navarra \\
\hline GD4 & $\begin{array}{l}\text { Seis mujeres y } \\
\text { dos hombres }\end{array}$ & $\begin{array}{l}\text { Conservas de frutas y hortali- } \\
\text { zas. Verduras congeladas y } \\
\text { empaquetado de verduras } \\
\text { frescas. Empresas con conve- } \\
\text { nio colectivo propio. }\end{array}$ & Navarra \\
\hline GD5 & Once mujeres & $\begin{array}{l}\text { Cultivo y recolección de toma- } \\
\text { tes y flores. Manipulado del } \\
\text { tomate. }\end{array}$ & Canarias \\
\hline GD6 & Tres mujeres & Conservas de pescado & Galicia \\
\hline GD7 & $\begin{array}{l}\text { Tres hombres y } \\
\text { dos mujeres }\end{array}$ & Industria láctea & Cantabria \\
\hline GD8 & $\begin{array}{l}\text { Siete mujeres y } \\
\text { un hombre }\end{array}$ & Conservas de pescado & Cantabria \\
\hline
\end{tabular}

\section{Bibliografía}

Acker, J. (1989). Doing Comparable Worth. Gender, Class and Pay Equity. Filadelfia: Temple University Press.

Acker, J. (1990). Hierarchies, jobs, bodies: a theory of gendered organizations. Gender and Society. Vol. 4 Núm. 2, 139-158.

Acker, J. (2006). Inequality regimes. Gender and Society. Vol. 20 Núm. 4, 441-464. 
Adkins, L. y Lury, C. (1999). The labour of identity: performing identities, performing economies. Economy and Society. Vol. 28 Núm. 4, 598-614.

Armstrong, P. y Cornish, M. (1997). Restructuring pay equity for a restructured work force: Canadian perspectives. Gender, Work and Organization. Vol. 4 Núm. 2, 67-86.

Armstrong, M., Cummins, A., Hastings, S. y Wood, W. (2007). Job Evaluation. A guide to achieving equal pay. Londres: Kogan Page.

Babiano, J. (2007). Mujeres, trabajo y militancia laboral bajo el franquismo (materiales para un análisis histórico). En J. Babiano, (Ed.), Del hogar a la huelga. Trabajo, género y movimiento obrero durante el franquismo (pp. 25-76). Madrid: Catarata.

Banks, M. y Milestone, K. (2010). Individualization, gender and cultural work. Gender, Work and Organization. Vol. 18 Núm. 1, 73-89.

Beechey, V. (1990/1994). Género y trabajo. Replanteamiento de la definición del trabajo. En C. Borderías, C. Carrasco y C. Alemany, (Eds.), Las mujeres y el trabajo (pp. 425-450). Barcelona: Icaria-Fuhem.

Belt, V., Richardson, R. y Webster, J. (2002). Women, social skill and interactive service work in telephone call centres. New Technology, Work and Employment. Vol. 17 Núm. 1, 20-34.

Booth, A. L., Francesconi, M. y Frank, J. (2003). A sticky floor model of promotion, pay and gender. European Economic Review. Vol. 47, 407-27.

Brynin, M. (2006). Gender, technology and jobs. British Journal of Sociology. Vol. 57 Núm. 3, 437-453.

Caprile, M. y Serrano Pascual, A. (2011). The move towards the knowledge-based society: a gender approach. Gender, Work and Organization. Vol. 18 Núm. 1, 48-72.

Callejo Gallego, J. (2005). Percepción de cambios en los tiempos sociales. Ponencia presentada en las jornadas Tiempos, actividades, sujeto. Una mirada desde la perspectiva de género. Madrid, 18 de febrero, Escuela de Relaciones Laborales.

Callejo Gallego, J. (2001). El grupo de discusión: introducción a una práctica de investigación. Barcelona: Ariel.

Cockburn, C. (1981). The material of male power. Feminist Review. Vol. 9, 41-58. 
Cockburn, C. (1983). Brothers. Male dominance and technological change. Londres: Pluto Press.

Cockburn, C. (1992). Abriendo la caja negra: la tecnología en los análisis de la sociología feminista. Sociología del Trabajo. Vol. 15, 91-107.

Cockburn, C. (1991). In the way of women: men's resistance to sex equality in organizations. Londres: Macmillan.

Comisión Europea (1994). Memorandum of equal pay for work of equal value. $\operatorname{COM}(94) 6$ final.

Comisión Europea (2007). Comunicación de la Comisión al Consejo, al Parlamento Europeo, al Comité Económico y Social Europeo y al Comité de las Regiones. Actuar contra la diferencia de retribución entre mujeres y hombres. COM (2007) 424 final.

Comisión Europea (2009). Opinion on the effectiveness of the current legal framework on equal pay for equal work or work of equal value in tackling the gender pay gap. Bruselas: Employment, Social Affairs and Equal Opportunities.

Daune-Richard, A. (2005). La cualificación en la sociología francesa: en busca de las mujeres. En J. Laufer, C. Marry y M. Maruani, (Eds.), El trabajo del género. Las ciencias sociales ante el reto de las diferencias de sexo (pp. 173-190). Valencia: Germania.

Eveline, J. y Todd, P. (2008). Gender mainstreaming: the answer to the gender pay gap? Gender, Work and Organization. Vol. 16 Núm. 5, 536-558.

Fernández Gómez, J. A. (2003). El peonaje femenino en la industria de material eléctrico y electrónico durante el franquismo. Sociología del Trabajo. Vol. 47, invierno, 43-73.

Flecker, J., Meil, P. y Pollert, A. (1998). The sexual division of labour in process manufacturing: economic restructuring, training and 'women's work'. European Journal of Industrial Relations. Vol. 4 Núm.1, 7-34.

Frenkel, M. (2008). Reprogramming femininity? The construction of gender identities in the Israeli hi-tech industry between global and local gender orders. Gender, Work and Organization. Vol. 15 Núm. 4, 352-374.

Glucksmann, M. (1986). In a class of their own? Women workers in the new industries in inter-war Britain. Feminist Review. Vol. 24 otoño, 7-37. 
Hart, S. M. (2002). The pay equity bargaining process in Newfoundland: understanding cooperation and conflict by incorporating gender and class. Gender, Work and Organization. Vol. 9 Núm. 4, 355-371.

Hartman, H. (1976/1994). Capitalismo, patriarcado y segregación de los empleos por sexos. En J. Laufer, C. Marry y M. Maruani, (Eds.), El trabajo del género. Las ciencias sociales ante el reto de las diferencias de sexo (pp. 253-294). Valencia: Germania.

Hollander, J. A. (2002). Resisting vulnerability: the social reconstruction of gender in interaction. Social Problems. Vol. 49 Núm. 4, 474-496.

Joo Kee, H. (2006). Glass ceiling or sticky floor? Exploring the Australian gender pay gap. The Economic Record. Vol. 82, 408-427.

Jones, D. y Torrie, R. (2009). Entering the twilight zone: the local complexities of pay and employment equity in New Zealand. Gender, Work and Organization. Vol. 16 Núm. 5, 559-578.

Joshi, J., Makepeace, G. y Dolton, P. (2007). More or less unequal? Evidence on the pay of men and women from the British cohort studies. Gender, Work and Organization. Vol. 14 Núm. 1, 37-55.

Kergoat, D. (2003). De la relación social de sexo al sujeto sexuado. Revista Mexicana de Sociología. Vol. 65 Núm. 4, 841-861.

Laufer, J., Marry, C. y Maruani, M. (2005). Introducción. En J. Laufer, C. Marry y M. Maruani, (Eds.), El trabajo del género. Las ciencias sociales ante el reto de las diferencias de sexo (pp. 19-34). Valencia: Germania.

Lindsay, S. (2008). The care-tech link: an examination of gender, care and technical work in healthcare sector. Gender, Work and Organization. Vol. 15 Núm. 4, 333351.

Litttler, C. (1982). The development of the labour process in capitalist societies. Londres: Heinemann.

Littler, C. (1990). The labour process: a theoretical review 1974-88. En D. Knights y H. Wilmot, (Eds.), Labour process theory (pp. 46-94). Hampshire: Macmillan.

Marini, M. (1989). Sex differences in earnings in the United States. Annual Review of Sociology. Vol. 15, 343-380.

Maruani, M. (1991). La construcción social de las diferencias de sexos en el mercado de trabajo. Revista de Economía y Sociología del Trabajo. Vol. 13-14, pp. 129-137. 
Maruani, M. (1993). La cualificación, una construcción social sexuada. Revista de Economía y Sociología del Trabajo. Vol. 21-22, 41-50.

Michon, F. (2005). Segmentación, mercados profesionales, mercados transicionales: la desaparición de las divisiones de género. En J. Laufer, C. Marry y M. Maruani, (Eds.), El trabajo del género. Las ciencias sociales ante el reto de las diferencias de sexo (pp. 297-322). Valencia: Germania.

Miles, B. M. y Huberman, A. M. (1994) Qualitative data análisis. An expanded sourcebook. $2^{\mathrm{a}}$ edición. Londres: Sage Publications.

Moore, S. (2007). Age as a factor defining older women's experience of labour market participation in the UK. Industrial Law Journal. Vol. 36 Núm. 3, 383-387.

Morillo Balado, R. (2006). Sistema de clasificación profesional y salarios. En R. Escudero, (Ed.), La negociación colectiva en España: una mirada crítica (pp. 437-448). Valencia: Tirant lo Blanch.

Móssesdóttir, L. (2011). Gender (in)equalities in the knowledge society. Gender, Work and Organization. Vol. 18 Núm. 1, 30-47.

OIT (Organización Internacional del Trabajo) (2008). Gender-neutral job evaluation for equal pay. Ginebra: OIT.

Parlamento Europeo (2002). Resolución sobre igual salario por trabajo de igual valor. Diario Oficial C 77 E de 28 de marzo.

Peng, P. y Singh, P. (2009). Pay equity in Ontario: the case of a non-profit senior services organization. Canadian Public Administration. Vol. 52 Núm. 4, 613625 .

Peterson, H. (2007). Gendered work ideals in Swedish IT firms: valued and not valued workers. Gender, Work and Organization. Vol. 14 Núm. 4, 333-348.

Philips, A. y Taylor, B. (1980). Sex and skill: notes towards a feminist economics. Feminist Review. Vol. 6, 79-88.

Quintanilla Navarro, B. (1996). Discriminación retributiva. Madrid: Marcial Pons.

Reich, M., Gordon, D. M. y Edwards, R. C. (1973). A theory of labor market segmentation. American Economics Review. Vol. 63 Núm. 2, 359-65.

Rubery, J., Grimshaw, D. y Fingered, H. (2005). How to close the gender pay gap in Europe: towards the gender mainstreaming of pay policy. Industrial Relations Journal. Vol. 36 Núm. 3, 184-213. 
Rudin, J. P. y Byrd, K. (2003). U.S. pay equity legislation: sheep in wolves' clothing. Employee Responsibilities and Rights Journal. Vol. 15 Núm. 4, 183-190.

Saldaña Valderas, E. (2004). Discriminación retributiva en función del género. Consejo Asesor de Relaciones Laborales. Sevilla: Mergablum.

Scholarios, D. y Taylor, P. (2010). Gender, choice and constraint in call centre employment. New Technology, Work and Employment. Vol. 25 Núm. 2, 101-116.

Simpson, R. (2011). Masculinity at work: the experiences of men in female dominated occupations. Work, Employment and Society. Vol. 18 Núm. 2, 349-368.

Smith, M. (2008). Gender, pay and work satisfaction at a UK university. Gender, Work and Organization. Vol. 16, Núm. 5, 621-641.

Thompson, E. P. (1967/1979). Tiempo, disciplina de trabajo y capitalismo industrial. En E. P. Thompson Tradición, revuelta y consciencia de clase. Barcelona: Editorial Crítica.

Vallés, M. (1999) Técnicas cualitativas de investigación social. Madrid: Síntesis Sociología.

Wajcman, J. (2000). Feminism facing industrial relations in Britain. British Journal of Industrial Relations. Vol. 38 Núm. 2, 183-201.

Walby, S. (2011). Is the knowledge society gendered? Gender, Work and Organization. Vol. 18 Núm. 1, 1-29.

Warren, T. (2007). Conceptualizing breadwinning work. Work, Employment and Society. Vol. 21 Núm. 2, 317-336.

West, J. (1990). Gender and the labour process: a reassessment. En D. Knights y H. Willmott, (Eds.), Labour process theory (pp. 245-273). Londres: Heinemann.

Whitehouse, G., Zeitlin, D. y Earnshaw, J. (2001). Prosecuting pay equity: evolving strategies in Britain and Australia. Gender, Work and Organization. Vol. 8 Núm. 4, 365-386.

Whitehouse, G. (2003). Gender and pay equity: future research directions. Asia Pacific Journal of Human Resources. Vol. 41 Núm. 1, 116-128.

Zarapuz, L. (2009). Las diferencias salariales entre hombres y mujeres en la industria de la alimentación. C.S. de CC.OO. Mimeo. Madrid. 